Office of Naval Research

Department of the Navy

Contract Nonr-220(35)

\title{
GENERAL FORMULATION OF A PERTURBATION THEORY FOR UNSTEADY CAVITY FLOWS
}

By

D. P. Wang and T. Yao-tsu Wu

Hydrodynamics Laboratory

Kármán Laboratory of Fluid Mechanics and Jet Propulsion

California Institute of Technology

Pasadena, California 


\section{ABSTRACT}

The problem of a two-dimensional cavity flow of an ideal fluid with small unsteady disturbances in a gravity free field is considered. By regarding the unsteady motion as a small perturbation of an established steady cavity flow, a fundamental formulation of the problem is presented. It is shown that the unsteady disturbance generates a surface wave propagating downstream along the free cavity boundary, much in the same way as the classical gravity waves in water, only with the centrifugal acceleration owing to the curvature of the streamlines in the basic flow playing the role of an equivalent gravity effect. As a particularly simple example, the surface waves in a hollow potential vortex flow is calculated by using the present theory. 


\section{INTRODUCTION}

Since the pioneering contributions of Helmholtz $(1)^{1}$ and Kirchhoff ${ }^{(2)}$, nearly a century ago, on the subject of steady, irrotational, plane flows involving free streamlines, extensive applications have been made to jets and to flows with a cavity or wake formation. In spite of such a long his tory and the mature state of steady free streamline theory and its wide applications to engineering problems, the subject of unsteady cavity flows has received attention only in the last seventeen years or so. Some of the difficulties involved in unsteady cavity flows can be envisaged as follows. The theoretical treatment of irrotational, two-dimensional cavitating flows of an ideal fluid is usually based on a certain proposed physical model, for example, the Kirchhoff-Helmholtz model. If the flow is steady, the exact solution of such a problem, within the assumption of the proposed model, is usually obtained by using the hodograph method, since in this case a surface of constant pressure is also one of constant speed. This property, however, no longer holds valid in the case when the flow is unsteady. Consequently, in order to investigate some of the characteristics of unsteady cavitating flows, different approaches and approximations have been introduced by various authors. Some of the early contributions have been dis cussed by Gilbarg ${ }^{(3)}$, Birkhoff and Zarantonello(4). In order to help appraise the present state of the knowledge, a brief survey may be made here of the recent developments.

1 Numbers in parenthesis refer to similarly numbered references in bibliography at end of paper. 
In 1949 von Kármán ${ }^{(5)}$ treated an accelerated flow normal to a flat plate held fixed in an inertial frame such that with a certain acceleration, the flow separates from the plate to form a closed cavity of constant shape attached behind the plate, and he obtained a solution for a particular Froude number characterizing the acceleration. The entire set of flows for cavities with constant shape was later derived by Gilbarg ${ }^{(6)}$ for arbitrary polygonal obstacles. For cavities with varying shape, Gilbarg ${ }^{(6)}$ proposed the as sumption that the free boundary, which is a material line, may be approximated by a streamline. As pointed out by Gilbarg, it seems physically reasonable that errors from this approximation may be quite small, at least for not too rapidly varying flows. Adopting this approximation, Woods ${ }^{(7)}$ treated the unsteady cavitating flow past curved obstacles with a finite cavity closed in the rear by a second fictitious body, as in the Riabouchinsky model for steady cavity flows ${ }^{(3)}$. Noticing the essential difference between the two distinct cases when the fluid at infinity is accelerating or when the body is accelerating (in an inertial frame), $\mathrm{Yih}^{(8)}$ treated both cases, deriving general formulae for unsteady cavity flows when the velocity potential $\Phi$ assumes the form $\Phi=U(t) f(x, y)$.

For the general case of unsteady cavity flows, another approach is to regard the unsteady part of the motion as a small perturbation of a steady cavity flow already established. With this approach Ablow and Hayes (9) developed a perturbation theory which was later employed by Fox and Morgan ${ }^{(10)}$ to investigate the stability problem of some free sur- 
face flows. Also, Curle $\mathrm{f}^{(1)}$ considered the large and small time solutions of a jet issuing from a slit. In these perturbation theories, the exact, linearized boundary conditions on the free surface are used. In this category a somewhat different perturbation theory has been applied to several specific problems by Woods ${ }^{(12)}$, Parkin ${ }^{(13)}, \mathrm{Wu}^{(14)}$, Timman (15) and Geurst $(16,17)$. In these latter works the free surface of an unsteady cavity flow is approximated by a streamline, thus releasing completely the kinematic condition imposed on the free boundary. By doing so, it is hoped that such approximation can give satisfactory results, perhaps for slowly varying flows. Based on such an approximation the resulting flow has been interpreted ${ }^{(12)}$ to contain the effect that an unsteady disturbance applied on the solid body will produce two vortex sheets leaving the separation points, propagating downstream on the free surface of the cavity with a velocity equal to that of the free stream of the basic flow. On physical grounds it can perhaps be argued that the linearized theory based on the approximation that the material lines be replaced by streamlines would become less consistent and less accurate for moderately and rapidly varying flows. On the other hand, the approach of Ablow and Hayes seems to have not yet been fully extended to treat the general case of unsteady cavity flows. It is the purpose of the present work to present a consistent formulation of a perturbation theory for the general case, following a method rather independent of that of Ablow and Hayes.

By assuming the time-dependent part of the flow to be small, a perturbation theory is developed here by a systematic linearization in the physical plane, without assuming that the displaced free surface of the 
cavity be approximated by a streamline. From this general formulation it is seen that the unsteady motion of the solid body produces in general free surface dynamic waves propagating along the cavity boundary, much the same as the gravity waves generated by a floating body in motion. The centrifugal force owing to the curvature of the streamlines in the basic flow now plays the role of an equivalent gravity in the classical water wave problem. In this sense, the unsteady cavity flows are similar in nature to the radiation of gravity waves over a flat water surface, only now in a much more complex form since the centrifugal acceleration varies along the cavity surface. Such a dynamic wave phenomenon cannot be found in the theory using the streamlineapproximation mentioned previously. A simple illustration of the present formulation is carried out for the surface waves over a hollow vortex first treated by Lord Kelvin ${ }^{(18)}$. Numerical results of typical unsteady cavity flows by using the present theory generally involve extensive analytical details; such results will be presented in a later work. It is the hope of this paper to stimulate further interest in developing this important and interesting subject, and in making applications for engineering purposes.

\section{GENERAL THEORY}

We suppose that for the time $t<0$ a steady, irrotational, twoaımensional flow past a solid body has been established (in a gravityfree field), its solution being assumed to be known. For $t>0$ the solid bodv is given an unsteady small disturbance, whose magnitude is char- 
acterized by a small parameter $\epsilon$. The resulting flow will be assumed to remain ir rotational in a region containing the body-cavity system. We shall establish a perturbation theory, to the first order in $\epsilon$, by regarding the time-dependent part of the flow as a small perturbation of the basic steady flow.

Under this assumption the flow possesses a velocity potential $\varphi(\mathrm{x}, \mathrm{y}, \mathrm{t} ; \epsilon)$ which may be expanded for $\mathrm{t}>0$ as

$$
\varphi(x, y, t ; \epsilon)=\varphi_{0}(x, y)+\epsilon \varphi_{1}(x, y, t)+0\left(\epsilon^{2}\right) .
$$

where $x, y$ are the Cartesian coordinates of the physical plane, $\varphi_{0}(x, y)$ is the velocity potential of the basic steady flow, $\varphi_{1}(x, y, t)$ is the perturbation potential, being independent of $\epsilon$. It may be noted that in the present formulation the space variables $(x, y)$ are not perturbed. Strictly speaking, the function $\psi_{\mathrm{o}}(\mathrm{x}, \mathrm{y})$ is defined only at points within the region of the basic steady flow, whereas $\varphi(x, y, t ; \epsilon)$ may exist at points outside that region as dictated by the perturbed flow configuration. Under such circumstances it is assumed that the basic flow potential $\varphi_{\mathrm{O}}(\mathrm{x}, \mathrm{y})$ may be continued analytically into the region wherever needed. It is clear that $\varphi(\mathrm{x}, \mathrm{y}, \mathrm{t} ; \epsilon), \varphi_{\mathrm{O}}(\mathrm{x}, \mathrm{y})$ and $\varphi_{1}(\mathrm{x}, \mathrm{y}, \mathrm{t})$ are all harmonic functions of $\mathrm{x}, \mathrm{y}$. We may further introduce the complex variable $\mathrm{z}=\mathrm{x}+\mathrm{iy}$, the complex potential $f=\varphi+i \psi$, and the complex velocity $w=u-i v$, defined by:

$$
w=\frac{d f}{d z}=q e^{-i \theta}
$$

with $q$ denoting the velocity magnitude and $\theta$ the flow inclination with the positive $x$-axis. The coresponding expansions of $f$ and $w$ are 


$$
\begin{aligned}
& f(z, t ; \epsilon)=f_{0}(z)+\epsilon f_{1}(z, t)+0\left(\epsilon^{2}\right), \\
& w(z, t ; \epsilon)=w_{0}(z)+\epsilon w_{1}(z, t)+0\left(\epsilon^{2}\right) .
\end{aligned}
$$

Here $f_{0}(z)=\varphi_{0}(x, y)+i \psi_{0}(x, y)$ is the complex potential of the basic flow, $f_{1}(z, t)=\varphi_{1}(x, y, t)+i \psi_{1}(x, y, t)$ is the complex perturbation potential, both being analytic functions of $z$. .

The pressure $p$ is given by the Bernoulli equation

$$
\frac{p}{\rho}+\frac{\partial \varphi}{\partial t}+\frac{1}{2}(\nabla \varphi)^{2}=C
$$

where $\rho$ is the constant fluid density, $C$ may be a function of $t$ only, which, after being absorbed by the term $\partial \varphi / \partial t$, may be taken a constant. Consistent with the above perturbation scheme, $p$ is written in the form

$$
p(x, y, t ; \epsilon)=p_{0}(x, y)+\epsilon p_{1}(x, y, t)+O\left(\epsilon^{2}\right)
$$

The pressure $p_{0}$ of the basic flow satisfies the steady form of (5).

$$
\frac{1}{\rho} \mathrm{p}_{\mathrm{O}}+\frac{1}{2}\left(\nabla \varphi_{\mathrm{O}}\right)^{2}=\frac{1}{\rho} \mathrm{p}_{\infty}+\frac{1}{2} \mathrm{U}^{2}=\frac{1}{\rho} \mathrm{p}_{\mathrm{c}}+\frac{1}{2} \mathrm{q}_{\mathrm{c}}{ }^{2}
$$

where $p_{\infty}$, $U$ are respectively the free stream pressure and velocity, $\mathrm{p}_{\mathrm{C}}$ the constant cavity pressure, $\mathrm{q}_{\mathrm{C}}$ the constant flow speed on the cavity boundary of the basic flow, which is characterized by the cavitation number $\sigma$ defined by

$$
\sigma \equiv\left(p_{\infty}-p_{c}\right) /\left(\frac{1}{2} \rho U^{2}\right)=\left(q_{c} / U\right)^{2}-1 \text {. }
$$

To facilitate the subsequent analysis, it is convenient to introduce a set of intrinsic coordinate $(s, n)$ as an alternative space variable, 
where $s$ is the arc length measured along a streamline in the direction of the basic flow, and $n$ the distance measured normal to a streamline in the direction of increasing $\psi_{0}$, as shown in Figure 1. Thus, the functions $s\left(\varphi_{0}, \psi_{0}\right), \mathrm{n}\left(\varphi_{0}, \psi_{0}\right)$ can be defined by

$$
\mathrm{d} \varphi_{\mathrm{O}}=\mathrm{q}_{\mathrm{O}}\left(\varphi_{0}, \psi_{\mathrm{O}}\right) \mathrm{ds}, \quad \mathrm{d} \psi_{\mathrm{O}}=\mathrm{q}_{\mathrm{O}}\left(\varphi_{0}, \psi_{0}\right) \mathrm{dn},
$$

with ds measured along $\psi_{0}=$ constant and $d n$ along $\varphi_{0}=$ constant. Consequently, the differentiations with respect to $\mathrm{s}$ and $\mathrm{n}$ are defined as

$$
\frac{\partial}{\partial s}=q_{0}\left(\frac{\partial}{\partial \varphi_{0}}\right){ }_{\psi_{0}} \quad \frac{\partial}{\partial n}=q_{0}\left(\frac{\partial}{\partial \psi_{0}}\right) .
$$

In terms of $(s, n)$, the continuity equation and ir rotationality condition become respectively

$$
\begin{aligned}
& \frac{\partial q_{0}}{\partial s}+q_{0} \frac{\partial \theta}{\partial n}=0, \\
& \frac{\partial q_{0}}{\partial n}-q_{0} \frac{\partial \theta}{\partial s}=0 .
\end{aligned}
$$

The boundary conditions of this problem are as follows:

(i) There are two boundary conditions on the free surface of the cavity, one being kinematic and the other dynamic in nature. Let the displacement of the perturbed free surface of the cavity, $S_{f}$, from that of the steady basic flow, $S_{f}$, be denoted by 


$$
F(s, n, t ; \epsilon)=n-\epsilon h(s, t)=0,
$$

so that $S_{f}$ is given by $n=0$ (see Figure 1 ). Then the kinematic condition that the fluid particules on the free surface will remain on it requires

$$
\frac{\mathrm{DF}}{\mathrm{Dt}}=\frac{\partial \mathrm{F}}{\partial \mathrm{t}}+\frac{\partial \varphi}{\partial \mathrm{s}} \frac{\partial \mathrm{F}}{\partial \mathrm{s}}+\frac{\partial \varphi}{\partial \mathrm{n}} \quad \frac{\partial \mathrm{F}}{\partial \mathrm{n}}=0 \quad \text { on } \quad \mathrm{n}=\epsilon \mathrm{h}(\mathrm{s}, \mathrm{t})
$$

By noting the definition of $s\left(\varphi_{0}, \psi_{0}\right), \mathrm{n}\left(\varphi_{0}, \psi_{0}\right)$ given by $(9)$, one finds from (11) that

$$
\frac{\partial F}{\partial n}=1-\epsilon \frac{\partial h}{\partial s}\left(\frac{\partial s}{\partial n}\right)_{\varphi_{0}}, \quad \frac{\partial F}{\partial s}=\left(\frac{\partial n}{\partial s}\right)_{\psi_{0}}-\epsilon \frac{\partial h}{\partial s} .
$$

Also from (9) one readily derives that

$$
\frac{\partial n}{\partial s}=q_{0}\left(\frac{\partial n}{\partial \varphi_{0}}\right)_{\psi_{0}}=q_{0} \int_{0}^{\psi_{0}} \frac{\partial}{\partial \varphi_{0}}\left(\frac{1}{q_{0}}\right) d \psi_{0}=q_{0} \int_{0}^{n} \frac{\partial}{\partial s}\left(\frac{1}{q_{0}}\right) d n ;
$$

and similarly,

$$
\frac{\partial \dot{s}}{\partial n}=q_{0} \int_{0}^{s} \frac{\partial}{\partial n}\left(\frac{1}{q_{0}}\right) d s
$$

Substituting (13), (14) into (12), and using the expansion (1), one obtains, up to the order $\epsilon$,

$$
\frac{\partial \varphi_{1}}{\partial n}=\frac{\partial h}{\partial t}+q_{0} \frac{\partial h}{\partial s}+h \frac{\partial q_{0}}{\partial s} \text { on } n=\epsilon h(s, t),
$$

in which use has been made of the general relationship $\partial \varphi_{0} / \partial s=q_{0}$, and $\partial \varphi_{\mathrm{O}} / \partial \mathrm{n}=0$. After expanding the quantities in (15) about the undisturbed free surface, or $n=0$, it is obvious that the same expression as above holds valid on $\mathrm{n}=0$. Now, by further applying the boundary condition that on $n=0, q_{o}=q_{c}$, which is a constant, the kinematic condition 
finally becomes

$$
\frac{\partial \varphi_{1}}{\partial n}=\frac{\partial h}{\partial t}+q_{c} \frac{\partial h}{\partial s} \text { on } \quad n=0
$$

For the dynamic condition, we assume here that the perturbed cavity boundary is subject to a prescribed unsteady, but uniform pressure perturbation,

$$
p(s, n, t ; \epsilon)=p_{c}+\epsilon p_{1}^{*}(t) \quad \text { on } \quad n=\epsilon h(s, t) \text {. }
$$

Substituting (17) and (1) into the Bernoulli equation (5), and using the property $\partial \varphi_{0} / \partial n=0$, one finds

$$
\epsilon \frac{1}{\rho} \mathrm{p}_{1}^{*}(\mathrm{t})+\epsilon \frac{\partial \varphi_{1}}{\partial t}+\frac{1}{2}\left(\frac{\partial \varphi_{\mathrm{o}}}{\partial \mathrm{s}}\right)^{2}+\epsilon \frac{\partial \varphi_{\mathrm{o}}}{\partial \mathrm{s}} \frac{\partial \varphi_{1}}{\partial \mathrm{s}}+0\left(\epsilon^{2}\right)=\frac{1}{2} \mathrm{q}_{\mathrm{c}}^{2} \text { on } \mathrm{s}_{\mathrm{f}}^{\prime}
$$

Expanding various quantities in the above equation about $n=0$, and using $\left(\partial \varphi_{\mathrm{o}} / \partial \mathrm{s}\right)=\mathrm{q}_{\mathrm{c}}$ on $\mathrm{n}=0$, one obtains, up to the order $\epsilon$,

$$
\frac{\partial \varphi_{1}}{\partial t}+q_{c}\left(\frac{\partial \varphi_{1}}{\partial s}+h \frac{\partial q_{0}}{\partial n}\right)=-\frac{1}{\rho} p_{1}^{*}(t) \text { on } n=0
$$

Now, from the irrotationality condition (10b) for the basic flow,

$$
\left(\frac{\partial q_{0}}{\partial n}\right)_{n=0}=\left(q_{0} \frac{\partial \theta}{\partial s}\right)_{n=0}=\mp \frac{q_{c}}{R}
$$

where $R$ is the radius of curvature of the steady cavity boundary, the - (or +) sign holds for the upper (or lower) branch of the cavity wall. These signs are necessary to make $R$ always a positive quantity. For a steady cavity flow it is as sumed that the cavity pressure is a minimum pressure in the flow field, which implies that the cavity surface of the basic flow is concave when viewed from the cavity, hence $\partial \theta / \partial s$ is negative on the 
upper cavity boundary AI and positive on the lower boundary BI (cf. Figure 1). The first order dynamic condition is therefore

$$
\frac{\partial \varphi_{1}}{\partial t}+\mathrm{q}_{\mathrm{c}} \frac{\partial \varphi_{1}}{\partial \mathrm{s}} \mp \frac{\mathrm{q}_{\mathrm{c}}^{2}}{\mathrm{R}} \mathrm{h}=-\frac{1}{\mathrm{p}} \mathrm{p}_{1}^{*}(\mathrm{t}) \quad \text { on } \quad \mathrm{n}=0 .
$$

Equations (16) and (18) are two conditions on the cavity free surface; they can be combined ịto one for $\varphi_{1}$ by eliminating $h$, giving

$$
\frac{1}{\mathrm{R}} \mathrm{D}\left(\mathrm{RD} \varphi_{1}\right) \mp \frac{\mathrm{q}_{\mathrm{c}}{ }^{2}}{\mathrm{R}} \frac{\partial \varphi_{1}}{\partial \mathrm{n}}=-\frac{1}{\rho}\left(\frac{\mathrm{R}^{\prime}}{\mathrm{R}} \mathrm{p}_{1}^{*}+\frac{\mathrm{dp}}{\mathrm{dt}}\right) \text { on } \mathrm{n}=0 \text {, }
$$

where

$$
D \equiv \frac{\partial}{\partial t}+q_{c} \frac{\partial}{\partial s^{\prime}} \quad R^{\prime} \equiv d R / d s
$$

At this point it is of interest to note that if $\mathrm{q}_{\mathrm{C}}^{2} / \mathrm{R}$ is regarded as an equivalent gravitational acceleration $g$ and the $s$-coordinate is rectilinear, then (16) and (18), or equivalently (19), are in the same form as those boundary conditions in the classical water wave problems in a gravity field, with $g$ pointing towards the interior of the flow. Thus, the centrifugal acceleration $\mathrm{q}_{\mathrm{C}}{ }^{2} / \mathrm{R}$ due to the curvature of the basic flow streamline now plays the role of the restoring force, much the same as gravity in water waves, in producing and propagating the surface waves along the curved cavity boundary. It may be remarked here that, if the perturbed free surface is approximated by its unperturbed steady free streamline boundary, thereby releasing the kinematic condition (16) and also the term with $h$ in (18), then the essential restoring force is altogether dropped out. On physical grounds, it may therefore be expected that the present formulation will yield result fundamentally-different 
from a theory using the approximation that the perturbed free surface be replaced by a streamline.

The free surface condition (19) may also be expressed in a complex variable form. First we note from (4) that on $S_{f}$ of the basic flow,

$$
\theta=\mathrm{i} \log \left(\mathrm{w}_{\mathrm{o}} / \mathrm{q}_{\mathrm{c}}\right)
$$

Hence,

$$
\frac{1}{\mathrm{R}}=\mp \frac{\partial \theta}{\partial \mathrm{s}}=\mp i \frac{1}{\mathrm{w}_{\mathrm{o}}} \frac{\partial \mathrm{w}_{\mathrm{o}}}{\partial \mathrm{s}}=\mp i \frac{\mathrm{q}_{\mathrm{c}}}{\mathrm{w}_{\mathrm{o}}} \frac{\partial \mathrm{w}_{\mathrm{o}}}{\partial \varphi_{\mathrm{o}}}=\mp i \frac{\mathrm{q}_{\mathrm{c}}}{\mathrm{w}_{\mathrm{o}}} \frac{\mathrm{dw}_{\mathrm{o}}}{\mathrm{df} \mathrm{f}_{\mathrm{o}}}
$$

For compactness, we impose the normalization that $\mathrm{q}_{c}=1$. Then, by using $(9 \mathrm{~b})$ and the Cauchy-Riemann equation $\partial \varphi_{1} / \partial \psi_{0}=-\partial \psi_{1} / \partial \varphi_{0}$, can be finally written as

$$
\begin{aligned}
& \operatorname{Re}\left[\left\{\left(\frac{\partial}{\partial t}+\frac{\partial}{\partial f_{0}}\right)^{2}-\left[\frac{d}{d f_{0}} \log \left(\frac{1}{w_{0}} \frac{d w_{o}}{d f_{0}}\right)\right]\left(\frac{\partial}{\partial t}+\frac{\partial}{\partial f_{0}}\right)-\left(\frac{1}{w_{0}} \frac{d w_{o}}{d f_{0}}\right) \frac{\partial}{\partial f_{0}} f_{1}\left(f_{0}, t\right)\right]\right. \\
& =\frac{-1}{\rho}\left[\mathrm{p}_{1}^{*} \frac{\mathrm{d}}{\mathrm{df}} \log \left(\frac{1}{\mathrm{w}_{\mathrm{o}}} \frac{\mathrm{dw}_{\mathrm{o}}}{\mathrm{df}}\right)+\frac{\mathrm{dp}_{\mathrm{o}}^{*}}{\mathrm{dt}}\right] \text { on } \mathrm{s}_{\mathrm{f}}
\end{aligned}
$$

which is the free surface condition in a complex variable form.

(ii) At the solid surface the normal component of the flow velocity relative to the moving boundary must vanish. Again using the intrinsic coordinates $(s, n)$, let the displacement of the wetted side of the solid body, $S_{0}^{\prime}$, from its basic position $S_{0}$ be prescribed by

$$
F(s, n, t ; \epsilon)=n-\epsilon h(s, t)=0 \text { on } s_{0}^{\prime}
$$

over a range of $s$ covered by the solid surface in the steady flow. Then it is clear that the kinematic condition (15) also holds valid-on the solid 
surface $S_{0}($ or $n=0)$, that is,

$$
\frac{\partial \varphi}{\partial \mathrm{n}}=\frac{\partial \mathrm{h}}{\partial \mathrm{t}}+\frac{\partial}{\partial \mathrm{s}}\left(\mathrm{q}_{\mathrm{O}} \mathrm{h}\right) \text { on } \mathrm{S}_{\mathrm{o}} \text {, }
$$

$h$ being here a known function of $s$ and $t$. The equivalent complex form of (22) is readily seen to be

$$
\operatorname{Im} \frac{\partial f_{1}\left(f_{o}, t\right)}{\partial f_{0}}=\frac{-1}{q_{0}}\left[\frac{\partial h}{\partial t}+\frac{\partial}{\partial s}\left(q_{0} h\right)\right] \text { on } s_{0}
$$

Note that the speed $q_{0}$ of the basic flow on the solid surface $S_{0}$ is not a constant.

(iii) The condition at the point at infinity depends on the free stream velocity and on whether or not the cavity volume is permitted to change with time. If the free stream velocity has a prescribed small perturbation $U_{1}(t) \quad\left(U_{1}\right.$ may be complex), so that the free stream velocity is $U_{\infty}=U+\epsilon U_{1}(t)$, then we require that

$$
w_{1}=\frac{\partial f}{\partial z} \rightarrow U_{1}(t) \text { as }|z| \rightarrow \infty
$$

Furthermore, when changes in the cavity volume are involved, then an appropriate representation of the flow can be made by introducing a fluid source at the point at infinity, as discussed by $\mathrm{Wu}^{(19)}$. For such cases, a finite cavity model for the basic flow is required to incorporate the source at infinity into the flow problem, as shown previously by the present authors $(20)$. These points have been further justified from a physical and mathematical ground by Benjamin (21). Furthermore, from Kelvin's theorem on the conservation of circulation, the circulation 
around the point at infinity cannot be changed in unsteady flow for $t<\infty$. Therefore, in addition to condition (24), it is required that

$$
\oint_{\Gamma} \frac{d f}{d z} d z=i Q_{1}(t)
$$

where $\Gamma$ is a contour around the point at infinity and $Q_{1}(t)$ is equal to the time rate of increase in cavity volume, which is supposed to be prescribed.

Finally, we state that if the problem is of the initial value type, then no radiation condition is needed for the surface waves; the se waves will turn out to propagate automatically towards downstream. However, in the case of simple harmonic motions, when being treated as a quasisteady flow, then the so-called "radiation condition" will be needed to ensure that the waves generated by body motion will not propagate upstream on the cavity surface. This completes our formulation of the problem.

\section{SURFACE WAVES ON A HOLLOW VORTEX}

This relatively simple problem was chosen to demonstrate an application of this general theory to a special case; this application is partly meant for a verification of the complicated expression of the free surface boundary condition, since the problem has already been solved by Lord Kelvin ${ }^{(18)}$ in a completely different way.

The basic flow is an irrotational, circulating motion about a point, say $z=0$, as center. The free surface will be denoted by 
$|z|=a$ on which the speed of flow is normalized to unity. The velocity potential is simply

$$
f_{0}(z)=-i a \log z
$$

and therefore,

$$
w_{0}(z)=-\frac{i a}{z}
$$

The transformation

$$
\zeta=i \frac{1-w_{0}}{1+w_{0}}
$$

maps the entire basic flow in the hodograph $w_{0}-$ plane, $\left|w_{0}\right| \leqslant 1$, into the upper half of the $\zeta$-plane (s ee figure 2).

We assume that the cavity pressure be kept at constant, that is, $p_{1}(t)=0$, then the boundary conditions of this problem are

$$
\operatorname{Re}\left[L\left(f_{1}\right)\right]=0 \quad \text { on } \quad \eta=0^{+}, \quad-\infty<\xi<\infty \text {, }
$$

where $L=\left(\frac{\partial}{\partial f_{0}}+\frac{\partial}{\partial t}\right)^{2}-\left\{\frac{d}{d f_{0}} \log \left(\frac{1}{w_{0}} \frac{d w_{o}}{d f_{0}}\right)\right\}\left(\frac{\partial}{\partial f_{0}}+\frac{\partial}{\partial t}\right)-\frac{1}{w_{o}} \frac{d w_{0}}{d f_{0}} \frac{\partial}{d f_{0}}$,

$$
\left|\frac{\partial f}{\partial z}\right|=0 \quad \text { as } \quad|z| \rightarrow \infty
$$

and

$$
\oint_{\Gamma}\left(\frac{\partial f}{\partial z}\right) d z=0
$$

where $\Gamma$ is a contour around the point at infinity, or, $\zeta=i$. A particular solution of the above boundary value problem is

$$
L\left(f_{1}\right)=0 \text {. }
$$

The complementary solution can be written as

$$
L\left(f_{1}\right)=\sum_{n=-\infty}^{\infty}\left[c_{n}(t)(\zeta-i)^{n}-\overline{c_{n}(t)}(\zeta+i)^{n}\right],
$$


where $c_{n}(t)$ are unknown functions of $t$. In order to satisfy equations (30) and (31), that is, $L\left(f_{1}\right)=O\left[(\zeta-i)^{a}\right], a>0$, as $\zeta \rightarrow_{i}$, and $\left|L\left(f_{1}\right)\right|<\infty$ at every point on the free surface it is necessary that $c_{n}=0$ for all $n$. Therefore, the solution of this boundary value problem is given by (32) alone.

By use of equations (26) and (27), equation (32) can be changed into

$$
\left(\frac{z^{2}}{a^{2}} \frac{\partial^{2}}{\partial z^{2}}+\frac{2 z}{a^{2}} \frac{\partial}{\partial z}-\frac{2 i}{a} z \frac{\partial^{2}}{\partial z \partial t}-\frac{\partial^{2}}{\partial t^{2}}\right) f_{1}(z, t)=0 .
$$

Since $f_{1}(z, t)$ should be regular everywhere outside $|z|=a$, we may write

$$
f_{1}(z, t)=\sum_{n=1,2}^{\infty} A_{n}(t) \frac{1}{z^{n}}
$$

Substitution of equation (35) into equation (34) gives

$$
\ddot{A}_{n}(t)-\frac{2 i n}{a} \dot{A}_{n}(t)-\frac{n}{a^{2}}(n-1) A_{n}(t)=0 \text {, }
$$

where dot represents the differentiation with respect to $t$. The solution of the last equation is

$$
A_{n}=a_{n} e^{\frac{i}{a}(n \pm \sqrt{n}) t}
$$

where $a_{n}$ are constants. Finally, we have

$$
f_{1}(z, t)=\sum_{n=1,2, \ldots}^{\infty}\left[a_{n} e^{\frac{i}{a}(n+\sqrt{n}) t}+\beta_{n} e^{\frac{i}{a}(n-\sqrt{n}) t}\right] \frac{1}{z^{n}},
$$


where the constants $a_{n}$ and $\beta_{n}$. can be determined by appropriate initial conditions. The above result agrees with that obtained by Lord Kelvin (18).

\section{ACKNOW LEDGMENT}

This work is sponsored in part by the Office of Naval Research of the U. S. Navy, under contract Nonr-220(35). Reproduction in whole or in part is permitted for any purpose of the United States Government. 


\section{BIB LIOGRAPHY}

1. Über discontinuierliche Flüssigkeitsbewegungen. Helmholtz, H., Monatsber. Berlin. Akad. 1868, pp. 215-228.

2. Zur Theorie freier Flüssigkeitsstrahlen. Kirchhoff, G., J. reine angew. Math. Vol. 70, 1869, pp. 289-298.

3. Jets and Cavities; Encyclopedia of Physics, Vol. IX. Gilbarg, D., Springer-Verlag, Berlin, Gottingen, Heidelberg, 1960, pp. 356363 ; pp. $321-326$.

4. Jets, Wakes and Cavities. Birkhoff, G. \& Zarantonello, E. H. , Academic Press, New York, 1957, pp. 236-257.

5. Collected Works of Theodore von Kármán, Vol. 4. von Kármán, T. , Betterworth Scientific Publications, London, 1956, pp. 396-398.

6. Unsteady Flow with Free Boundaries. Gilbarg, D., Zeitschrift für angewandte Mathematic und Physik, Vol. 3, 1952, pp. 34-42.

7. Unsteady Cavitating Flow Past Curved Obstacles. Woods, L. C., A. R. C. Technical Report C. P. No. 149, 1954.

8. Finite Two-Dimensional Cavities. Yih, C. S., Proc. Roy. Soc., London. Series A, Vol. 256, 1960, pp. 90-100.

9. Perturbation of Free Surface Flows. Ablow, C. M. and Hayes, W. D. , Tech. Report 1, Division Applied Math., Brown Univ., 1951.

10. On the Stability of Some Flows of an Ideal Fluid with Free Surfaces. Fox, J. L. and Morgan, G. W., Quarterly of Applied Math., Vol. 11, 1954, pp. 439-456.

11. Unsteady Two-Dimensional Flows with Free Boundaries. Curle, N. , Proc. Roy. Soc., London. Series A, Vol. 235, 1956, pp. 375-395.

12. Unsteady Plane Flow Past Curved Obstacles with Infinite Wakes. Woods, L. C., Proc. Roy. Soc., London. Series A, Vol. 229, 1955, pp. $152-180$.

13. Fully Cavitating Hydrofoils in Nonsteady Motion. Parkin, B. R. , Engineering Division Report No. 85-2, Calif. Inst. of Tech., 1957.

14. A Linearized Theory for Nonsteady Cavity Flows. Wu, T. Y., Engineering Division Report No. 85-6, Calif. Inst. of Tech., 1957.

15. A General Linearized Theory for Cavitating Hydrofoils in Nonsteady Flow. Timman, R., Second Symposium, Naval Hydrodynamics, Wa shington, D. C. , U. S. A., 1958, pp. 559-579.

16. Unsteady Motion of A Flat Plate in A Cavity Flow. Geurst, J. A., Report No. 21, Inst. of Applied Math. , Technological Univ., Delft, Holland, 1959. 


\section{BIB LIOGRAPHY \\ (continued)}

17. A Linearized Theory for the Unsteady Motion of a Supercavitating Hydrofoil. Geurst, J. A., Report No. 22, Inst. of Applied Math., Technological Univ., Delft, Holland, 1960.

18. Vibration of a Columnar Vortex. Thompson, Wm., Philosophical Magazine, $10(5)$, 1880, pp. 155-168.

19. Unsteady Supercavitating Flows. Wu, T. Y., Second Symposium, Naval Hydrodynamics, Washington, D. C., U.S. A. , 1958, pp. 293-313.

20. Small Time Behavior of Unsteady Cavity Flows. Wang, D. P. and Wu, T. Y., Archive for Rational Mechanics and Analysis, Vol. 14, No. 2, 1963, pp. 127-152.

21. Note on the interpretation of two-dimensional theories of growing cavities, Benjamin, T. B., Journal of Fluid Mechanics, Vol. 19, 1964, pp. 137-144. 

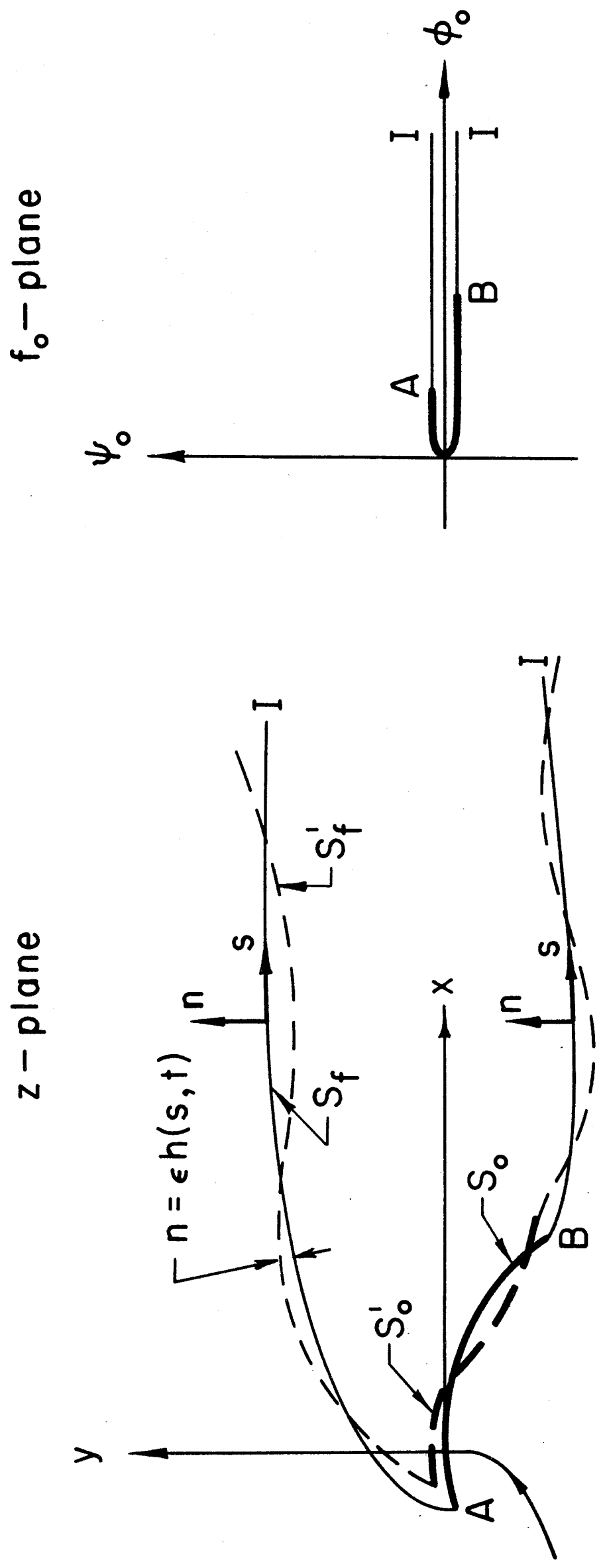

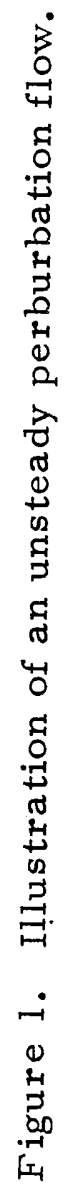



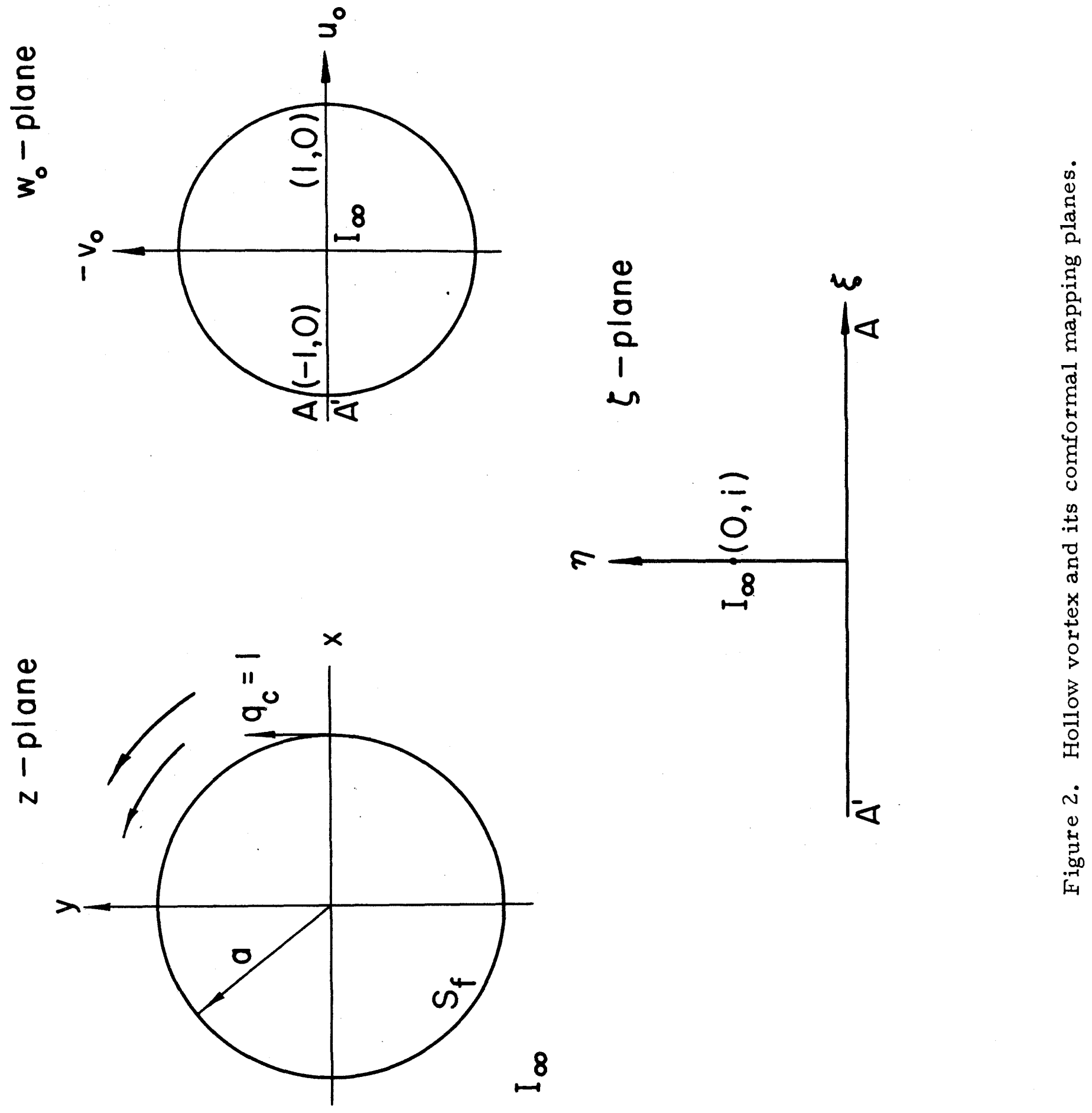
Chief of Naval Research Department of the Navy Washington 25, D. C. Attn: Codes 438 (3) 461 463 466

Commanding Officer Office of Naval Research Branch Office 495 Summer Street Boston 10, Mas sachusetts

Commanding Officer Office of Naval Research Branch Office 207 West 24th Street New York 11, New York Commanding Officer Office of Naval Research Branch Office 1030 East Green Street Pasadena, California

Commanding Officer Office of Naval Research Branch Office 1000 Geary Street San Francisco 9, California

Commanding Officer

Office of Naval Research Branch Office

Box 39, Navy No. 100

Fleet Post Office

New York, New York (25)

Director

Naval Research Laboratory

Washington 25, D. C.

Attn: Code 2027 (6)

Chief, Bureau of Naval Weapons Department of the Navy

Wa shington 25, D. C.

Attn: Codes RUAW-r RRRE

RAAD

RAAD-222

DIS -42
Commander

U. S. Naval Ordnance Test Station China Lake, California

Attn: Code 753

Chief, Bureau of Ships Department of the Navy Washington 25, D. C.

Attn: Codes 310

$$
\begin{aligned}
& 312 \\
& 335 \\
& 420 \\
& 421 \\
& 440 \\
& 442 \\
& 449
\end{aligned}
$$

Chief, Bureau of Yards and Docks Department of the Navy Washington 25, D. C.

Attn: Code D-400

Commanding Officer and Director David Taylor Model Basin

Washington 7, D. C.

Attn: Codes 108

142

500

513

520

525

526

$526 \mathrm{~A}$

530

533

580

585

589

591

$591 \mathrm{~A}$

700

Commander

U. S. Naval Ordnance Test Station Pasadena Annex 3202 E. Foothill Blvd.

Pasadena 8, California

Attn: Code P-508

Commander

Planning Department

Portsmouth Naval Shipyard

Portsmouth, New Hampshire 
Commander

Planning Department

Boston Naval Shipyard

Boston 29, Massachusetts

Commander

Planning Department

Pearl Harbor Naval Shipyard

Navy No. 128, Fleet Post Office

San Francisco, California

Commander

Planning Department

San Francisco Naval Shipyard

San Francisco 24, California

Commander

Planning Department

Mare Island Naval Shipyard

Vallejo, California

Commander

Planning Department

New York Naval Shipyard

Brooklyn 1, New York

Commander

Planning Department

Puget Sound Naval Shipyard

Bremerton, Washington

Commander

Planning Department

Philadelphia Naval Shipyard

U. S. Naval Base

Philadelphia 12, Pennsylvania

Commander

Planning Department

Norfolk Naval Shipyard

Portsmouth, Virginia

Commander

Planning Department

Charleston Naval Shipyard

U. S. Naval Base

Charleston, South Carolina

Commander

Planning Department

Long Beach Naval Shipyard

Long Beach 2, California

Commander

Planning Department

U. S. Naval Weapons Laboratory

Dahlgren, Virginia

Commander

U. S. Naval Ordnance Laboratory

White Oak, Maryland
Dr. A. V. Hershey

Computation and Exterior

Ballistics Laboratory

U. S. Naval Weapons Laboratory

Dahlgren, Virginia

Superintendent

U. S. Naval Aca demy

Annapolis, Maryland

Attn: Library

Superintendent

U. S. Naval Postgraduate School

Monterey, California

Commandant

U. S. Coast Guard

1300 E Street, N. W.

Washington, D. C.

Secretary Ship Structure Committee

U. S. Coast Guard Headquarters

1300 E Street, N. W.

Washington, D. C.

Commander

Military Sea Transportation Service Department of the Navy

Washington 25, D. C.

U. S. Maritime Administration

GAO Building

441 G Street, N. W.

Wa shington, D. C.

Attn: Division of Ship Design

Division of Research

Superintendent

U. S. Merchant Marine Academy

Kings Point, Long Island, New York

Attn: Capt. L. S. McCready

(Department of Engineering)

Commanding Officer and Director

U. S. Navy Mine Defense Laboratory

Panama City, Florida

Commanding Officer

NROTC and Naval Administrative

Massachusetts Institute of Technology

Cambridge 39, Massachusetts

U. S. Army Transportation Research

and Development Command

Fort Eustis, Virginia

Attn: Marine Transport Division

Mr. J. B. Parkinson

National Aeronautics and

Space Administration

$1512 \mathrm{H}$. Street, N. W.

Washington 25, D. C. 
Director

Langley Research Center

Langley Station

Hampton, Virginia

Attn: Mr. I. E. Garrick

Mr. D. J. Marten

Director Engineering Science Division National Science Foundation

1951 Constitution Avenue, N. W.

Wașhington 25, D. C.

Director

National Bureau of Standards

Wa shington 25, D. C.

Attn: Fluid Mechanics Division

(Dr. G. B. Schubauer)

Dr. G. H. Keulegan

Dr. J. M. Franklin

Defense Documentation Center

Camer on Station

Alexandria, Virginia

Office of Technical Services

Department of Commerce

Wa shington 25, D. C.

California Institute of Technology

Pasadena 4, California

Attn: Professor M. S. Plesset

Professor T. Y. Wu

Professor A. J. Acosta

University of California

Department of Engineering

Los Angeles 24, California

Attn: Dr. A. Powell

Director

Scripps Institute of Oceanography

University of California

La Jolla, California

Professor M. L. Albertson

Department of Civil Engineering

Colorado $\mathrm{A}$ and $\mathrm{M}$ College

Fort Collins, Colorado

Professor J. E. Cernak

Department of Civil Engineering

Colorado State University

Fort Collins, Colorado

Professor W. R. Sears

Graduate School of

Aeronautical Engineering

Cornell University

Ithaca, New York

State University of Iowa

Iowa Institute of Hydraulic Research

Iowa City, Iowa

Attn: Dr. H. Rouse

Dr. L. Landweber
Massachusetts Institute of Technology

Cambridge 39, Massachusetts

Attn: Department of Naval Architecture and Marine Engineering Professor A. T. Ippen

Harvard University

Cambridge 38, Massachusetts

Attn: Professor G. Birkhoff

(Department of Mathematics)

Professor G. F. Carrier

(Department of Mathematics)

University of Michigan

Ann Arbor, Michigan

Attn: Professor R. B. Couch (Department of Naval Architecture) Professor W. W. Willmarth (Department of Aeronautical Engineering)

Dr. L. G. Straub, Director

St. Anthony Falls Hydraulic Laboratory

University of Minnesota

Minneapolis 14, Minnesota

Attn: Mr. J. N. Wetzel

Professor B. Silberman

Professor J. J. Foody

Engineering Department

New York State University

Maritime College

Fort Schylyer, New York

New York University

Institute of Mathematical Sciences

25 Waverly Place

New York 3, New York

Attn: Professor J. Keller

Professor J. J. Stoker

The Johns Hopkins University

Department of Mechanical Engineering

Baltimore 18, Maryland

Attn: Professor S. Corrsin Profess or O. M. Phillips (2)

Massachusetts Institute of Technology

Department of Naval.Architecture and

Marine Engineering

Cambridge 39, Massachusetts

Attn: Professor M. A. Abkowitz

Dr. G. F. Wislicenus

Ordnance Research Laboratory

Pennsylvania State University

University Park, Pennsylvania

Attn: Dr. M. Sevik

Professor R. C. DiPrima

Department of Mathematics

Rensselaer Polytechnic Institute

Troy, New York - 
Director

Woods Hole Oceanographic Institute

Woods Hole, Massachusetts

Stevens Institute of Technology

Davidson Laboratory

Castle Point Station

Hoboken, New Jersey

Attn: Mr. D. Savitsky

Mr. J. P. Breslin

Mr. C. J. Henry

Mr. S. Tsakonas

Webb Institute of Naval Architecture

Crescent Beach Road

Glen Cove, New York

Attn: Professor E. V. Lewis

Technical Library

Executive Director

Air Force Office of Scientific Research

Washington 25, D. C.

Attn: Mechanics Branch

Commander

Wright Air Development Division

Aircraft Laboratory

Wright-Patterson Air Force Base, Ohio

Attn: Mr. W. Mykytow,

Dynamics Branch

Cornell Aeronautical Laboratory

4455 Genesee Street

Buffalo, New York

Attn: Mr. W. Targoff

Mr. R. White

Massachusetts Institute of Technology

Fluid Dynamics Research Laboratory

Cambridge 39, Massachusetts

Attn: Professor H. Ashley

Professor M. Landahl

Professor J. Dugundji

Hamburgische Schiffbau-Versuchsanstalt

Bramfelder Strasse 164

Hamburg 33, Germany

Attn: Dr. H. Schwanecke

Dr. H. W. Lerbs

Institut fur Schiffbau der

Univer sitat Hamburg

Berliner Tor 21

Hamburg 1, Germany

Attn: Professor G. P. Weinblum

Transportation Technical Research Institute 1-1057, Mejiro-Cho, Toshima-Ku

Tokyo, Japan

Max-Planck Institut fur Stromungsfors-

chung Bottingerstrasse 6/8

Gottingen, Germany

Attn: Dr. H. Reichardt
Hydro-og Aerodynamisk Laboratorium

Lyngby, Denmark

Attn: Professor Carl Prohaska

Skipsmodelltanken

Trondheim, Norway

Attn: Professor J. K. Lunde

Versuchsanstalt fur Wasserbau and

Schiffbau

Schleuseninsel im Tiergarten

Berlin, Germany

Attn: Dr. S. Schuster, Director

Dr. Grosse

Technische Hogeschool

Institut voor Toegepaste Wiskunde

Julianalaan 132

Delft, Netherlands

Attn: Professor R. Timman

Bureau D'Analyse et de Recherche

Appliquees

47 Avenue Victor Bresson

Is sy-Les-Moulineaux

Seine, France

Attn: Professor Siestrunck

Netherlands Ship Model Basin

Wageningen, The Netherlands

Attn: Dr. Ir. J. D. van Manen

National Physical Laboratory

Teddington, Middlesex, England

Attn: Mr. A. Silverleaf, Superintendent Ship Division Head, Aerodynamics Division

Head, Aerodynamics Department Royal Aircraft Establishment Farnborough, Hants, England Attn: Mr. M. O. W. Wolfe

Dr. S. F. Hoerner

148 Busteed Drive

Midland Park, New Jersey

Boeing Airplane Company

Seattle Division

Seattle, Washington

Attn: Mr. M. J. Turner

Electric Boat Division

General Dynamics Corporation

Groton, Connecticut

Attn: Mr. Robert McCandliss

General Applied Sciences Labs., Inc. Merrick and Stewart Avenues

Westbury, Long Island, New York

Gibbs and Cox, Inc.

21 West Street

New York, New York 
Lockheed Aircraft Corporation Missiles and Space Division

Palo Alto, California

Attn: R. W. Kermeen

Grumman Aircraft Engineering Corp. Bethpage, Long Island, New York

Attn: Mr. E. Baird

Mr. E. Bower

Mr. W. P. Carl

Midwest Research Institute 425 Volker Blvd.

Kansas City 10 Missouri

Attn: Mr. Zeydel

Director, Department of Mechanical

Sciences

Southwest Research Institute

8500 Culebra Road

San Antonio 6, Texas

Attn: Dr. H. N. Abramson

Mr. G. Ransleben

Editor, Applied Mechanics

Review

Convair

A Division of General Dynamics

San Diego, California

Attn: Mr. R. H. Oversmith

Mr. H. T. Brooke

Hughes Tool Company

Aircraft Division

Culver City, California

Attn: Mr. M. S. Harned

Hydronautics, Incorporated

Pindell School Road

Howard County

Laurel, Maryland

Attn: Mr. Phillip Eisenberg

Rand Development Corporation

13600 Deise Avenue

Cleveland 10, Ohio

Attn: Dr. A. S. Iberall

U. S. Rubber Company

Research and Development Department Wayne, New Jersey

Attn: Mr. L. M. White

Technical Research Group, Inc.

Route 110

Melville, New York, 11749

Attn: Mr. Jack Kotik

Mr. C. Wigley

Flat 102

6-9 Charterhouse Square

London, E. C. 1, England
AVCO Corporation

Lycoming Division

1701 K Street, N. W.

Apt. No. 904

Washington, D. C.

Attn: Mr. T. A. Duncan

Mr. J. G. Baker

Baker Manufacturing Company

Evansville, Wisconsin

Curtiss -Wright Corporation Research

Division

Turbomachinery Division

Quehanna, Pennsylvania

Attn: Mr. George H. Pedersen

Dr. Blaine R. Parkin

AiResearch Manufacturing Corporation

9851-9951 Sepulveda Boulevard

Los Angeles 45, California

The Boeing Company

Aero-Space Division

Seattle 24, Washington

Attn: Mr. R. E. Bateman

Internal Mail Station 46-74

Lockheed Aircraft Corporation

California Division

Hydrodynamics Research

Burbank, California

Attn: Mr. Bill East

National Research Council

Montreal Road

Ottawa 2, Canada

Attn: Mr. E. S. Turner

The Rand Corporation

1700 Main Street

Santa Monica, California

Attn: Technical Library

Stanford University

Department of Civil Engineering

Stanford, California

Attn: Dr. Byrne Perry

Dr. E. Y. Hsu

Dr. Hirsh Cohen

IBM Research Center

P. O. Box 218

Yorktown Heights, New York

Mr. David Wellinger

Hydrofoil Projects

Radio Corporation of America

Burlington, Massachusetts

Food Machinery Corporation

P. O. Box 367

San Jose, California

Attn: Mr. G. Tedrew 
Dr. T. R. Goodman

Oceanics, Inc.

Technical Industrial Park

Plainview, Long Island, New York

Professor Brunelle

Department of Aeronautical Engineering

Princeton, New Jersey

Commanding Officer

Office of Naval Research

Branch Office

230 N. Michigan Avenue,

Chicago 1, Illinois

University of Colorado

Aerospance Engineering Sciences

Boulder, Colorado

Attn: Professor M. S. Uberoi

The Pennsylvania State University

Department of Aeronautical

Engineering

Ordnance Research Laboratory

P. O. Box 30

State College, Pennsylvania

Attn: Professor J. William Holl

Institut fur Schiffbau der

Univer sitat Hamburg

Lammersieth 90

2 Hamburg 33, Germany

Attn: Dr. O. Grim

Technische Hogeschool

Laboratorium voor Scheepsbounkunde

Mekelweg 2, Delft, The Netherlands

Attn: Professor Ir. J. Gerritsma 